\title{
INTRODUÇÃO DE ESPÉCIES EM ECOSSISTEMAS AQUÁTICOS: CAUSAS, PREVENÇÃO E MEDIDAS DE CONTROLE
}

\author{
Sybelle Bellay* \\ Rafael Rogério Rosa* \\ André Nogueira Bozza* \\ Sue Ellen Prata Fernandes* \\ Márcio José Silveira**
}

RESUMO: Espécies não nativas causam danos imprevisíveis sobre os ecossistemas e são consideradas pela IUCN como a segunda maior causa mundial de perda de biodiversidade. Os ecossistemas aquáticos são particularmente vulneráveis a esse impacto e muitas pesquisas mostram que essas espécies não nativas podem alterar a composição das comunidades nativas. A proposta deste estudo é apresentar as principais causas de introduções de espécies em ecossistemas aquáticos e possíveis medidas de prevenção e controle de espécies invasoras. Em ambientes aquáticos destacam-se como causas de introduções: destruição de barreiras geográficas como construções de reservatórios e canais de transposição de rios; criação e melhoria dos estoques naturais, devido à falta de planejamento na construção e manutenção dos locais de criação; aquariofilia e ornamentação de paisagens. Além disso, o turismo através da pesca esportiva, o controle biológico na tentativa de exterminar outra espécie já introduzida, a fauna associada a qual é transportada junto com a água, substratos ou hospedeiros e água de lastro principalmente proveniente de navios mercantis, também contribuem para as introduções de espécies. Medidas de controle de espécies não nativas são escassas e a identificação de vetores, monitoramento, políticas públicas e estudos são algumas estratégias que podem ajudar a diminuir os impactos ambientais causados por espécies não nativas em ecossistemas aquáticos. A divulgação e orientação sobre os impactos ambientais causados por espécies não nativas direcionadas para a população leiga e científica faz-se necessário, sendo a Internet uma ferramenta imprescindível e prioritária no combate a espécies não nativas.

PALAVRAS-CHAVE: Ambiente Aquático; Espécies não Nativas; Impacto Ambiental; Invasão.

Programa de Pós-Graduação em Ecologia de Ambientes Aquáticos Continentais (PEA), Universidade Estadual de Maringá (UEM), Brasil. E-mail: sybellebellay@yahoo.com.br.

** Programa de Pós-Graduação em Biologia Comparada (PGB), Universidade Estadual de Maringá (UEM), Brasil. 


\title{
INTRODUCTION OF SPECIES IN AQUATIC ECOSYSTEMS: CAUSES, PREVENTION AND CONTROL
}

\begin{abstract}
Non-native species may cause great damage to ecosystems. IUCN considers them as the second major cause worldwide for the loss of biodiversity. Water ecosystems are highly vulnerable to the impact and several research works have demonstrated that non-native species may alter the composition of native communities. Current paper provides the main causes for the introduction of species in water ecosystems and possible prevention and control measures against invading species. The main causes of their introduction in water environments are the destruction of geographic barriers, such as the construction of reservoirs and transposition canals in rivers; the establishment and improvement of natural stocks due to lack of planning and maintenance of breeding sites; aquarium and ornamentation of landscape. Tourism represented by sport fishing, biological control to exterminate the previous introduced species, associated fauna transported together with the water, substrates or hosts and waste water from the merchant navy also contribute for the introduction of species. The control of non-native species is rare and the identification of vectors, monitoring, public policies and studies are strategies that may decrease the environmental impacts caused by non-native species in aquatic ecosystems. Orientation and instructions on the environmental impact by non-native species should be provided for the population. The Internet may be an important tool against non-native species.
\end{abstract}

KEY WORDS: Aquatic Environment; Non-Native Species; Environmental Species; Invasion.

\section{INTRODUÇÃO}

Relatos sobre biologia de invasões foram observados por Charles Darwin em seu clássico livro On the origen of species (1859), onde já mostrava exemplos sobre espécies não nativas e invasões biológicas. Entretanto, o primeiro cientista a escrever um livro voltado ao assunto foi Charles Elton - Ecology of invasions by animals and plants (1958), reconhecido como o ponto de partida para os estudos sobre invasões biológicas, difundindo o interesse por estudos sistemáticos com esse enfoque. Recentemente esse é um tema central da ecologia teórica e aplicada, abrangendo aspectos multidisciplinares (RICHARDSON; PYSEK, 2007). 
São inúmeros os casos de espécies que alcançaram áreas onde naturalmente não eram encontradas através de introduções (VITOUSEK et al., 1997). As espécies que foram deslocadas de suas áreas nativas são conhecidas de diversas formas (e.g., introduzidas, exóticas, alóctones, não indígenas, alienígenas ou não nativas) (COLAUTTI; MACISAAC, 2004) e provocam danos imprevisíveis sobre os ecossistemas receptores, podendo afetar tanto a biodiversidade quanto atividades econômicas (SOUZA et al., 2009). Aqui adotaremos o termo "espécie não nativa", por acreditarmos que este seja o termo menos controverso, e os casos analisados neste trabalho são referentes a espécies estabelecidas, visto que distorções nas interpretações das terminologias empregadas são muitas vezes utilizadas para amenizar os cenários das introduções (AGOSTINHO et al., 2007).

Os danos de espécies não nativas são graves e atualmente considerados pela União Internacional para a Conservação da Natureza (International Union for Conservation of Nature - IUCN) como a segunda maior causa de perda da biodiversidade, atrás apenas da destruição de habitat (RODRÍGUEZ, 2001). Em um primeiro momento, o sucesso das espécies não nativas sobre as nativas pode ser contraditório, visto que as últimas são evolutivamente especializadas ao ambiente (SAX; BROWN, 2000). Entretanto, o processo de invasões pode ser dividido em fases, nas quais existem barreiras que precisam ser superadas pela espécie não nativa (BLACKBURN et al., 2011). Dentre estas fases para uma invasão bem sucedida estaria a transposição de barreiras geográficas e de barreiras de cativeiro ou criação de espécimes, a sobrevivência no ambiente com uma reprodução bem sucedida, ausência de predadores e parasitos, aclimatação mais rápida a mudanças das condições ambientais, rápida adaptação genotípica ao ambiente, generalidade na utilização dos recursos e pressão de propágulos (SAX; BROWN, 2000; VITULE et al., 2009). No caso de ambientes aquáticos, a conexão entre corpos de água proporciona um canal importante para a dispersão das espécies não nativas (HAVEL et al., 2005).

Em um estudo desenvolvido por Lowe et al. (2000) foram apresentadas as 100 espécies mais invasivas do mundo. Dentre essas espécies, aproximadamente 25 estão relacionadas ao ambiente aquático, sendo evidente a participação de invertebrados (por exemplo, mexilhão dourado), peixes (por exemplo, Salmo trutta Linnaeus, 1758), "truta arco-íris" (Lates niloticus Linnaeus, 1758), "perca do Nilo" e plantas aquáticas como Eichbornia crassipes Solms-Laub, "aguapé", uma 
espécie da América do Sul com alta taxa de crescimento populacional, encontrada em mais de 50 países nos cinco continentes.

Este trabalho de revisão trata sobre as principais causas de introduções de espécies em ambientes aquáticos, as quais podem ser decorrentes de ações humanas diretas e/ou propositais em que uma espécie é levada de uma área a outra de forma intencional, ou deslocamentos de espécies decorrentes de ações humanas indiretas e não intencionais nas quais pode ser incluída, por exemplo, a mescla de fauna entre bacias hidrográficas conectadas por uma obra de transposição, apresentando casos de introdução de organismos aquáticos e seus efeitos deletérios sobre o ecossistema. Além disso, são abordadas medidas de prevenção e controle sobre a dispersão dessas espécies invasoras.

\section{AS PRINCIPAIS CAUSAS DAS INTRODUÇÕES EM AMBIENTES AQUÁTICOS}

Embora a conservação do ambiente não seja um assunto recente, as introduções em todo o mundo ainda continuam de modo acidental ou deliberado. A seguir são abordadas algumas formas e exemplos de introduções em ambientes aquáticos.

\subsection{DESTRUIÇÃO DE BARREIRAS GEOGRÁFICAS}

A construção de reservatórios e canais de transposição de rios prejudica a biodiversidade local, uma vez que expõem as espécies nativas a novas condições, tanto pelas alterações hidrológicas, como por alterações nas interações bióticas ao possibilitar que espécies de bacias hidrográficas distintas passem a interagir, assim como espécies de trechos isolados de uma mesma bacia (MOREIRA-FILHO; BUCKUP, 2005). Um exemplo evidente de introdução de espécies como consequência de uma barragem é o caso do reservatório de Itaipu, incluso na bacia do alto Rio Paraná, localizado na fronteira entre Brasil e Paraguai. Anteriormente à sua construção, duas províncias ictiofaunística distintas, a do médio Rio Paraná e a do alto Rio Paraná possuíam como barreira natural o Salto de Sete Quedas (BONETTO, 1986). Com a 
inundação desta barreira no enchimento do lago da usina hidrelétrica de Itaipu em 1982, houve uma notável dispersão de espécies do médio Paraná para os trechos a montante (AGOSTINHO et al., 1997), fato agravado posteriormente com a formação em 2002 do Canal da Piracema.

Segundo Graça e Pavanelli (2007), cerca de 30 espécies de peixes do médio Rio Paraná já foram detectadas no alto Rio Paraná em função direta da formação do reservatório ou do Canal de Piracema. Vitule et al. (2012), em seu estudo, demonstraram que cerca de 40 a 52\% das espécies atualmente presentes na planície de inundação do alto Rio Paraná dispersaram para essa região após a construção da Hidrelétrica de Itaipu. Além da degradação ambiental, foram detectadas nesse ambiente uma variabilidade na disponibilidade de recursos, alterações nas dinâmicas populacionais e padrões de dominâncias de espécies não nativas sobre as nativas (OPORTO, 2008).

Havel et al. (2005) sumarizaram características de reservatórios que podem influenciar a invasão de espécies aquáticas, principalmente microorganismos, sendo elas: distúrbio na paisagem, eutrofização, salinidade e alterações na cadeia trófica. Além dessas características dos reservatórios, transporte a longa ou baixa distância por humanos, conectividade com a paisagem através de corpos hídricos e transporte por vento ou aves são processos que regulam a invasão passiva de espécies exóticas em reservatórios.

\subsection{MANEJO E MELHORIA DOS ESTOQUES NATURAIS}

A falta de planejamento de produtores na construção e manutenção dos locais destinados ao manejo de espécies não nativas é um dos principais fatores relacionados com os escapes de criadouros, sendo frequentes as introduções destas espécies no ambiente (SAX; BROWN, 2000). A ruptura de tanques mal estruturados durante o período de chuvas tem contribuído relevantemente com a introdução de espécies exóticas em várias bacias (MILI; TEIXEIRA, 2006). Várias espécies podem ilustrar esta situação, tais como os bagres: Clarias gariepinus (Burchell, 1822) e Clarias batrachus (Linnaeus, 1758). Estas espécies nativas da África e Ásia foram introduzidas acidentalmente por escapes ou solturas por criadores comerciais ou 
domésticos em muitas regiões. Potencialmente invasivas estas espécies de bagre podem deixar a água rastejando com a ajuda das nadadeiras peitorais em busca de alimento ou sítio de reprodução, invadindo outros corpos d'água e ameaçando espécies nativas (MILI; TEIXEIRA, 2006; VITULE et al., 2006). Em vários reservatórios torna-se comum o uso de tanques-rede para aquicultura. De acordo com Ramos et al. (2005), além dos impactos ambientais diretamente relacionados à ictiofauna, tanques-rede quando mal empregados podem causar impactos na qualidade da água (eutrofização), alteração de sedimentos com consequências na comunidade bentônica e introdução de patógenos.

No Brasil, além de Cyprinus carpio (Linnaeus, 1758), "carpa", introduzida em todo o mundo e considerada uma espécie chave e engenheira de ecossistemas, pois altera o habitat devido ao comportamento forrageiro, consumindo plantas e revolvendo o substrato (GISD, 2012), propagação de parasitos, predar e competir com outras espécies por alimento (QUEROL et al., 2005), grande parte das espécies cultivadas foi introduzida de outros países ou continentes, tais como Oreochromis niloticus (Linnaeus, 1758) e Tilapia rendalli (Boulenger, 1897), "tilápias", Oncorbynchus mykiss (Walbaum, 1792), "truta-arco-íris", e bagres do gênero Clarias (DELARIVA; AGOSTINHO, 1999). Já as espécies Astronotus ocellatus (Agassiz, 1831), "apaiari", Cichla spp., "tucunaré", e Plagioscion squamosissimus (Heckel, 1840), "corvina” (DELARIVA; AGOSTINHO, 1999) são exemplos de espécies que foram introduzidas nas regiões Centro e Sul do Brasil, a partir da Amazônia ou do Nordeste (AGOSTINHO et al., 2007). Após a introdução dessas espécies, alguns estudos evidenciaram alterações em guildas tróficas através de predação ou competição (BARROS et al., 2012), bem como na riqueza de espécies nativas (LATINI; PETRERE, 2004).

As introduções de peixes em ambientes naturais visando aumentar o recurso pesqueiro e produção de lucros continua em ritmo acelerado (CASAL, 2006). Uma prática recorrente em diversos ambientes aquáticos é o peixamento (estocagem), onde milhares de juvenis são soltos em corpos d'água a fim de melhorar o recurso pesqueiro, entretanto falhas no processo de identificação das espécies a serem colocadas nestes locais podem desencadear uma série de danos ao ambiente (DALACORTE et al., 2009). Um exemplo clássico é o da introdução, em 1954, de Lates niloticus, "perca do Nilo", no lago Vitória pela administração colonial britânica que 
visava melhorar os recursos pesqueiros da região (AMNH, 2013). A introdução deste peixe está relacionada a vários casos de extinções, mudanças de dieta e declínio das populações de peixes locais. A extinção de aproximadamente 200 espécies endêmicas de ciclídeos haplochromídeos (WITTE et al., 1992), assim como a alteração da dieta de espécies de bagres e aves aquáticas que se alimentavam principalmente desses ciclídeos (GOUDSWAARD; WITTE, 1997), ocorreram direta ou indiretamente em função da competição ou predação de alguma fase do desenvolvimento da perca do Nilo, ou ainda em consequência das mudanças do habitat geradas pela deterioração do ecossistema a partir da exploração deste peixe (GOUDSWAARD et al., 2008).

\subsection{AQUARIOFILIA E ORNAMENTAÇÃO DE PAISAGENS}

Através do bobby da criação ou ato de ter animais de estimação, diversas espécies ganham novos locais de ocorrência a cada dia. Estima-se que mais de 60 milhões de pessoas em todo mundo cultivem peixes em aquários como passatempo (WHITTINGTON; CHISHOLM, 2008). A ocorrência do peixe Pterois volitans (Linnaeus, 1758), "lionfish", por exemplo, foi relatada na costa norte-americana do Oceano Atlântico e em torno do arquipélago das Bermudas no mar do Caribe desde o começo de 1990 (WHITFIELD et al., 2002). Essa espécie é nativa das águas tropicais do Pacífico Ocidental e a via mais provável de sua introdução ao longo da costa leste dos Estados Unidos é a soltura de aquários, entretanto a introdução por água de lastro não pode ser descartada (WHITFIELD et al., 2002). Esta espécie pode causar alterações deletérias em ecossistemas de recifes de coral através de predação de peixes e invertebrados, bem como a competição com predadores nativos (ALBINS; HIXON, 2008). Registrada atualmente em águas costeiras do Brasil, esta espécie tem sido indicada como uma das mais recentes ameaças para as espécies nativas (HADDAD et al., 2015).

Em relação às plantas aquáticas, muitas espécies foram introduzidas como ornamentação em aquários e hoje são protagonistas de grandes invasões causando prejuízos econômicos e ambientais através da obstrução de lagos e canais, entupimento das grades de proteção de turbinas em reservatórios, afetando a produção de energia elétrica, obstrução da pesca esportiva, alterações na qualidade da água e recreação (MARTINS et al., 2008). As piores invasões de macrófitas 
aquáticas em todo o mundo ocorreram pelas espécies E. crassipes, Myriophyllum aquaticum (Vell.), Salvinia molesta (DS Mitchell), Pistia stratiotes (L.) e Azolla filiculoides (Lamarck), distribuindo-se por vastas áreas e evidentes em muitos lagos e represas (WITTENBERG; COCK, 2001; GISD, 2012). A ausência de inimigos naturais ou predadores que exerçam o controle das populações de macrófitas introduzidas são importantes fatores que contribuem para a rápida expansão e potencial invasão (NEVES et al., 2002).

Nos últimos anos, uma nova espécie invasora foi identificada nas águas sul-africanas, a Hydrilla verticillata (L.F.) Royle, "hidrila", uma macrófita aquática submersa enraizada, nativa da Ásia (COOK; LUOND, 1982). Esta espécie também se tornou uma das principais plantas daninhas submersas, particularmente no sudeste dos EUA (BLACKBURN et al., 1969), e recentemente também observada em águas brasileiras (primeiros registros em 2005: THOMAZ et al., 2009a). Atualmente está disseminada em reservatórios e áreas naturais da bacia do Rio Paraná (THOMAZ et al., 2009b) e estudos nessa região têm demonstrado que a "hidrila" altera os fatores limnológicos, como oxigenação da água (POSEY et al., 1993) que, por sua vez, afetam a comunidade biológica residente, levando em casos extremos à morte de peixes e outros organismos aquáticos pela redução dos níveis de oxigênio na água (WITTENBERG; COCK, 2001).

\subsection{TURISMO}

O turismo pode apresentar uma contribuição significativa para a introdução de espécies (MCNEELY, 2001). Inúmeras embarcações se deslocam entre diferentes bacias, podendo levar incrustadas em seus cascos e/ou motores diversos organismos aquáticos, tais como invertebrados e propágulos de macrófitas. Em algumas regiões a pesca esportiva é desenvolvida tendo como base espécies introduzidas, um exemplo são os peixes do gênero Cichla, "tucunaré", que são muito apreciados neste tipo de pesca (GOMIERO; BRAGA, 2003). Além disso, diversas espécies de peixes como Erythrinus erythrinus (Bloch \& Schneider, 1801), Hoplerytbrinus unitaeniatus (Spix \& Agassiz, 1829) e todas as espécies do gênero Gymnotus são comercializadas e utilizadas como iscas vivas por pescadores, causando a introdução e distribuição 
dessas espécies em diversas bacias hidrográficas (JÚLIO JUNIOR et al., 2009).

\subsection{CONTROLE BIOLÓGICO}

Muitas vezes, para evitar o avanço de espécies não nativas e como uma opção substituta do uso de métodos químicos, é feita a introdução de espécies que são potencialmente capazes de controlar populações invasoras (THOMAZ, 2002). Peixes têm sido introduzidos para controle de macrófitas aquáticas nativas ou introduzidos em diversas regiões do mundo como, por exemplo, Ctenopharyngodon idella (Valenciennes, 1844), "carpa-capim”, que é originária de rios de planície da Rússia e China (THOMAZ, 2002; GOPALAKRISHNAN et al., 2011). O grande problema de introduzir espécies para controle biológico é que as mesmas podem se adaptar à utilização de recursos diferentes daquele proposto para o controle e passam a predar e competir e até mesmo extinguir espécies nativas, com mínima ação sobre as espécies-alvo (SIMBERLOFF; STILING, 1996).

\subsection{FAUNA ASSOCIADA}

Todo organismo introduzido pode ser o meio para que outras espécies sejam introduzidas. Juntamente com seus hospedeiros, parasitos e patógenos podem ser introduzidos e se estabelecer em novas áreas (DELARIVA; AGOSTINHO, 1999). Desta forma, diversos organismos de vida livre são transportados junto com a água de veículos especializados em transporte de peixes para aquicultura ou estocagem (peixamento), ou até mesmo nas raízes de plantas aquáticas que turistas possivelmente possam ter levado para ornamentação.

A aquicultura é responsável pelo caráter cosmopolita de alguns parasitos, como os crustáceos Lernaea cyprinacea (Linnaeus, 1758) e Argulus foliaceus (Linnaeus, 1758) (DELARIVA; AGOSTINHO, 1999). No Brasil, vários parasitos de peixes foram introduzidos em distintas bacias hidrográficas (GABRIELLI; ORSI, 2000). Espécies de crustáceos de Lernaea e Lamproglena são exemplos de parasitos que passaram a ocorrer em várias espécies de peixes no país após a introdução de Cyprinus carpio e Oreochromis niloticus, respectivamente, causando a mortandade de peixes e consequentes prejuízos econômicos (QUEROL et al., 2005). 
A introdução nos EUA de peixes como Lates niloticus e Oreochromis mossambicus (Peters, 1852) nativos da África pode ter proporcionado a introdução de Daphnia lumboltzi (Sars, 1885), cladócero nativo de regiões da África, Índia e Austrália (HAVEL; HEBERT, 1993). Este cladócero invasor de vida livre foi detectado nos EUA em 1991 (HAVEL; HEBERT, 1993), desde então vários outros registros foram observados para o continente americano, já estando presente na planície de inundação do alto Rio Paraná (SIMÕES et al., 2009).

\section{7 ÁGUA DE LASTRO}

$\mathrm{O}$ uso de água de lastro em grandes navios para conferir estabilidade e capacidade de manobra tem sido um meio eficaz de introdução de organismos marinhos e de água doce (OLIVEIRA et al., 2006). Os navios atuais transportam entre 06 e 10 bilhões de toneladas de água de lastro por ano, introduzindo espécies entre os continentes, com um deslocamento diário estimado de 3.000 a 7.000 espécies (SANTOS; LAMONICA, 2008). Por exemplo, levantamentos indicam que cerca de 370 espécies entre invertebrados marinhos e plantas já foram transportados corriqueiramente apenas entre portos japoneses e a costa oeste dos Estados Unidos através da água de lastro (SANTOS; LAMONICA, 2008).

Na América do Sul, uma espécie que tem causado bastante repercussão é Limnoperna fortunei (Dunker, 1857), "mexilhão dourado", que foi introduzida neste continente através da água de lastro de navios provenientes do sudeste da Ásia (China e Coreia) e registrado pela primeira vez em 1991 no Rio da Prata, próximo a Buenos Aires (PASTORINO et al., 1993). Após esta introdução acidental, L. fortunei tem se espalhado pela bacia do Rio da Prata, apresentando registros nas bacias de seus principais rios (Paraná, Paraguai, Uruguai e Tietê) (DARRIGRAN et al., 2012; ULIANOSILVA et al., 2013). Os registros em águas do Pantanal mostram que a espécie está se aproximando da bacia Amazônica, ameaçando desta forma a maior biodiversidade do mundo (ULIANO-SIIVA et al., 2013). A falta de competidores e predadores beneficia a rápida disseminação e colonização deste molusco tanto em substratos naturais (juncos, rochas), quanto artificiais, tais como boias, tubulações e cascos de navios (TORGAN et al., 2009). Além disso, o mexilhão dourado pode utilizar como substrato conchas de bivalves nativos, os quais podem ser sufocados pela presença 
do invasor aderido em um elevado número de indivíduos (DARRIGRAN; EZCURRA DE DRAGO, 2000). Limnoperna fortunei é conhecida por alterar paisagens, a fauna e flora aquática, além de acarretar prejuízos econômicos aos sistemas de geração de energia elétrica nas hidrelétricas e captação e tratamento de água por meio da formação de macroaglomerados compactos (DARRIGRAN; MANSUR, 2006).

\section{MEDIDAS DE PREVENÇÃO E CONTROLE}

Controlar a dispersão de espécies não nativas ou mesmo removê-las de ambientes invadidos muitas vezes torna-se uma atividade com altos custos financeiros e com baixa eficiência, porém evitar novas introduções e o monitoramento para que as espécies não nativas não aumentem suas áreas de ocorrência são medidas com alta relação custo-benefício (WITTENBERG; COCK, 2001). A identificação correta das espécies nativas de um determinado ambiente é um pré-requisito fundamental para conduzir as tentativas de controle das espécies não nativas, assim podendo estas ser reconhecidas no ambiente, verificando suas distribuições e abundâncias (SOUZA et al., 2009).

A conscientização dos riscos que as introduções de espécies podem ocasionar aos ecossistemas é o primeiro passo para a prevenção de novas introduções. Ao se reconhecer os riscos, a identificação de possíveis vetores na introdução de espécies pode tornar-se uma prática de maior frequência e necessária como medida de controle (SOUZA et al., 2009). Seguindo as principais causas de introduções em ambientes aquáticos abordadas no presente estudo podem-se elucidar algumas medidas de prevenção e controle:

- Políticas públicas que evitem a realização de obras que levem à conexão de bacias hidrográficas isoladas, assim como regióes dentro de uma mesma bacia que apresentem fauna e flora diferenciadas;

- Cuidados com a manutenção de tanques destinados para a produção de espécies não nativas, evitando escapes destes organismos para o ambiente;

- Conscientização de praticantes de aquariofilismo para que não soltem ou coloquem as espécies em ambientes onde possam ser 
potencialmente invasores;

- Instruir os turistas e pessoas que trabalham diretamente com turismo em ambientes aquáticos sobre a necessidade da limpeza dos equipamentos de pesca, assim como das embarcações para evitar que organismos aderidos ou presos nestes equipamentos sejam levados de um ambiente para outro possibilitando introduções de espécies;

- Realizar um estudo detalhado sobre a espécie indicada para ser utilizada na prática de controle biológico, pois a falta de conhecimentos sobre a biologia de espécies empregadas nessa prática podem ocasionar danos ainda mais prejudiciais ao ambiente do que a espécie que se pretende controlar;

- Para evitar que parasitos e patógenos sejam introduzidos juntos com seus hospedeiros em sistemas de criação e acidentalmente liberados no ambiente é importante que os novos indivíduos adquiridos pelos produtores passem um período em ambiente isolado (por exemplo, quarentena) e seja realizado o tratamento adequado assim que o parasito ou patógeno for detectado; e

- Buscar esterilizar a água de lastro e cumprir a Convenção Internacional para Controle e Gerenciamento da Água de Lastro e Sedimentos de Navios de 2004 criada pela International Maritime Organization (IMO).

\section{DISCUSSÃO}

Em todos os casos, após uma introdução, o controle sobre o avanço da espécie no ambiente está relacionado com o monitoramento, podendo ser aplicadas técnicas de remoção dos organismos, uso de substâncias químicas ou, como mencionado neste estudo, controle biológico. Em alguns países como o Brasil, programas de controle e erradicação de espécies introduzidas e invasoras ainda são raros, por necessitarem de elevados investimentos financeiros tanto para a pesquisa como para a realização das atividades de controle, mas o grande impasse é que não há garantia de que após serem tomadas medidas de controle e erradicação, a 
integridade biológica dos ambientes afetados possa se restabelecer (SOUZA et al., 2009).

Pesquisas sobre invasões biológicas têm sido tradicionalmente centradas nos componentes ecológicos das espécies não nativas, sem considerar ou por considerar de forma restrita, o conhecimento dos componentes sociais. A compreensão da visão humana sobre as invasões é fundamental para resolver os problemas associados às espécies não nativas (GARCÍA-LLORENTE et al., 2008). Segundo Larson et al. (2011), para ser sustentável, a gestão de espécies invasoras deve abordar os fatores ambientais, sociais e econômicos (ou "pilares") que influenciam as causas, impactos e controle de espécies não nativas em várias escalas espaciais e temporais. Embora esses pilares geralmente sejam reconhecidos pela população, a sua implementação é muitas vezes limitada por opções de controle insuficientes, restrições econômicas e políticas. A realização bem sucedida dos objetivos de qualquer um dos três pilares pode produzir feedbacks que ajudam a reforçar os outros pilares (LARSON et al., 2011). Entretanto, o descaso com o meio ambiente, visando apenas um retorno financeiro rápido ou a falta de compromisso com a manutenção da biodiversidade, presentes nas políticas governamentais de muitos países, podem ser entraves no avanço de medidas de controle contra novas introduções, pois diversas espécies cultivadas em todo o mundo foram introduzidas com financiamento e apoio governamentais, assim como obras de transposição de bacias e construção de hidrelétricas que facilitam essas introduções.

Questionários com usuários de ambientes (parques, por exemplo) que apresentam espécies não nativas devem ser feitos para medir seus conhecimentos, atitudes e preferências em relação a várias opções de gestão de espécies não nativas. Estes estudos podem informar práticas de gestão às espécies não nativas, fornecendo um quadro para a identificação de características das partes interessadas e sintetizar as preferências do público para minimizar conflitos e destacar os resultados ecologicamente benéficos e socialmente aceitáveis (SHARP et al., 2011).

\section{CONSIDERAÇÕES FINAIS}

O presente trabalho procurou demonstrar, de modo geral, como espécies não nativas aquáticas afetam o meio ambiente. Inúmeros são os exemplos dos danos 
que as introduções, invasões e estabelecimento de espécies podem ocasionar aos ecossistemas. Portanto, ainda faz-se necessário avançar nos métodos de prevenção e controle de espécies não nativas nos ambientes invadidos, bem como ações de divulgação e orientação para a população leiga quanto científica, sobre os impactos ambientais causados por essas espécies. O uso da Internet contribui para o aumento da divulgação de medidas de prevenção e controle de espécies não nativas, por disponibilizar conteúdos e o acesso às revistas especializadas no assunto, servir de repositório de informações, troca de conhecimento, bem como informações geográficas de distribuição dessas espécies, podendo auxiliar no controle de possíveis invasões, sendo uma ferramenta imprescindível e prioritária no combate a espécies não nativas.

\section{AGRADECIMENTOS}

Os autores são gratos ao PEA/CAPES-PROEX e CNPq pelo apoio logístico e financeiro.

\section{REFERÊNCIAS}

AGOSTINHO, A. A.; JÚLIO JR., H. F.; GOMES, L. C.; BINI, L M.; AGOSTINHO, C. S. Composição, abundância e distribuição espaço-temporal da ictiofauna. In: VAZZOLER, A. E. A. de M.; AGOSTINHO, A. A.; HAHN, N. S. (Org.) A Planície de Inundação do Alto Rio Paraná: aspectos físicos, biológicos e socioeconômicos. Maringá: EDUEM, 1997, p. 179-208.

AGOSTINHO, A. A.; GOMES, L. C.; PELICICE, F. M. Ecologia e manejo de recursos pesqueiros em reservatórios do Brasil. Maringá: EDUEM, 2007.

ALBINS, M. A.; HIXON, M. A. Invasive Indo-Pacific lionfish Pterois volitans reduce recruitment of Atlantic coral-reef fishes. Marine Ecology Progress Series, v. 367, p. 233-238, 2008. 
AMNH - THE AMERICAN MUSEUM OF NATURAL HISTORY. Lake Victoria. 2013.

Disponível em: <http://www.amnh.org/explore/resource-collections/biodiversitycrisis/lake-victoria >. Acesso em: 06 out. 2013.

BARROS, L. C.; SANTOS, U.; ZANUNCIO, J. C.; DERGAM, J. A. Plagioscion squamosissimus (Sciaenidae) and Parachromis managuensis (Cichlidae): A threat to native fishes of the Doce River in Minas Gerais, Brazil. PLOS ONE, v. 7, n. 6, 2012.

BLACKBURN, R. D.; WELDON, R. W.; YEO, R. R.; TAYLOR, T. M. Identification and distribution of certain similar-apperaing submerged aquatics weeds in Florida. Hyacinth Control Journal, v. 8, p. 17-21, 1969.

BLACKBURN, T. M.; PYSEK, P.; BACHER, S.; CARLTON, J. T.; RICHARD, P.; DUNCAN, R. P.; JAROSIK, V.; WILSON, J. U. W.; RICHARDSON, D. M. A proposed unified framework for biological invasions. Trends in Ecology and Evolution, v. 26, n. 7, p. 334-339, 2011.

BONETTO, A. A. The Paraná river system. In: DAVIES, B. R.; WALKER, K. F. (Org.) The Ecology of River Systems. Dordrecht: Dr. W. Junk Publishers, 1986, p. 541-555.

CASAL, C. M. V. Global documentation of fish introductions: the growing crisis and recommendations for action. Biological Invasions, v. 8, n. 1, p. 3-11, 2006.

COLAUTTI, R. I.; MACISAAC, H. J. A neutral terminology to define 'invasive' species. Diversity and Distributions, v. 10, p. 135-141, 2004.

COOK, C. D. K.; LUOND, R. A revision of the genus Hydrilla (Hydrocharitaceae). Aquatic Botany, v. 13, p. 485-504, 1982.

DALA-CORTE, R. B.; FRANZ I.; BARROS, M. P. de; OTT, P. H. Levantamento da ictiofauna da Floresta Nacional de Canela, na região superior da bacia hidrográfica do Rio Caí, Rio Grande do Sul, Brasil. Biota Neotropica, v. 9, n. 2, p. 221-226, 2009.

DARRIGRAN, G.; EZCURRA de DRAGO, I. Invasion of the exotic freshwater mussel Limnoperna fortunei (Dunker,1857) (Bivalvia: Mytilidae) in South America. Nautilus, v. 114, n. 2, p. 69-73, 2000. 
DARRIGRAN, G. E.; MANSUR, M. C. D. Distribuição, abundância e dispersão. In: DARRIGRAN, G. A.; DAMBORENEA, C. (Org.) Bio-invasion del mejillón dorado en el continente americano. La Plata: Edulp, La Plata, 2006, p. 93-110.

DARRIGRAN, G.; DAMBORENEA, C.; DRAGO, E. C.; EZCURRA de DRAGO, I.; PAIRA, A.; ARCHUBY, F. Invasion process of Limnoperna fortunei (Bivalvia: Mytilidae): The case of Uruguay River and emissaries of the Esteros del Iberá Wetland, Argentina. Zoologia, v. 29, n. 6, p. 531-539, 2012.

DARWIN, C. On the origin of species by means of natural selection, or the preservation of favoured races in the struggle for life. 1859. Disponivel em: < http://www.talkorigins.org/faqs/origin.html>. Acesso em: 06 out. 2013.

DELARIVA, R. L.; AGOSTINHO, A. A. Introdução de espécies: uma síntese comentada. Acta Scientiarum, v. 21, n. 2, p. 255-262, 1999.

ELTON, C. S. The ecology of invasions by animals and plants. London: The University of Chicago Press, Methuen, 1958.

GABRIELLI, M. A.; ORSI, M. L. Dispersão de Lernaea cyprinacea (Linnaeus) (Crustacea, Copepoda) na região norte do estado do Paraná, Brasil. Revista Brasileira de Zoologia, v. 17, n. 2, p. 395-399, 2000.

GARCÍA-LLORENTE, M.; MARTÍN-LÓPEZ, B.; GONZÁLEZ, J. A.; ALCORLO, P.; MONTES, C. Social perceptions of the impacts and benefits of invasive alien species: Implications for management. Biological Conservation, v. 141, p. 2969-2983, 2008.

GLOBAL INVASIVE SPECIES DATABASE - GISD. 2012. Disponível em: < http://www. issg.org/database/welcome/> . Acesso em: 16 out. 2013.

GOMIERO, L. M.; BRAGA, F. M. S. Pesca experimental do tucunaré, gênero Cichla (Osteichthyes, Cichlidae), no reservatório da UHE de Volta Grande, Rio Grande ( $48^{\circ}$ $25^{\prime}-47^{\circ} 35^{\prime}$ W, $19^{\circ} 57^{\prime}-20^{\circ} 10^{\prime}$ S). Boletim do Instituto de Pesca, v. 29, n. 1, p. 29$37,2003$.

GOPALAKRISHNAN, A.; RAJKUMAR, M.; SUN, J.; PARIDA, A.; VENMATHI MARAN, B. 
A. Integrated biological control of water hyacinths, Eichbornia crassipes by a novel combination of grass carp, Ctenopharyngodon idella (Valenciennes, 1844), and the weevil, Neochetina spp. Chinese Journal of Oceanology and Limnology, v. 29, n. 1, p. 162-166, 2011.

GOUDSWAARD, K .P. C.; WITTE, F. The catfish fauna of Lake Victoria after the Nile perch upsurge. Environmental Biology of Fishes, v. 49, p. 21-43, 1997.

GOUDSWAARD, K. P. C.; WITTE, F.; KATUNZI, E. F. B. The invasion of an introduced predator, Nile perch (Lates niloticus, L.) in Lake Victoria (East Africa): chronology and causes. Environmental Biology of Fishes, v. 81, n. 2, p. 127-139, 2008.

GRAÇA, W. J.; PAVANELLI, C. S. Peixes da planície de inundação do Alto Rio Paraná e áreas adjacentes. Maringá: EDUEM, 2007.

HADDAD, V.; STOLF, H. O.; RISK, J. Y.; FRANÇA, F. O.; CARDOSO, J. L. C. Report of 15 injuries caused by lionfish (Pterois volitans) in aquarists in Brazil: a critical assessment of the severity of envenomations. The Journal of Venomous Animals and Toxins Including Tropical Diseases, v. 21, n. 8, 2015.

HAVEL, J. E.; HEBERT, P. D. N. Daphnia lumboltzi in North America: Another exotic zooplankter. Limnology and Oceanography, v. 38, n. 8, p. 1823-1827, 1993.

HAVEL, J. E.; LEE, C. E.; ZANDEN, M. J. D. Do reservoirs facilitate invasion into landscapes? Bioscience, v. 55, n. 6, p. 518-525, 2005.

JÚLIO Jr., H. F.; DEL TÓS, C.; AGOSTINHO, A. A.; PAVANELLI, C. S. A massive invasion of fish species after eliminating a natural barrier in the upper Paraná River basin. Neotropical Ichthyology, v. 7, n. 4, p. 709-718, 2009.

LARSON, D. L.; PHILLIPS-MAO, L.; QUIRAM, G.; SHARPE, L.; STARK, R.; SUGITA, S.; WEILER, A. A framework for sustainable invasive species management: Environmental, social, and economic objectives. Journal of Environmental Management, v. 92, p. 14-22, 2011.

LATINI, A. O.; PETRERE Jr., M. Reduction of a native fish fauna by alien species: 
an example from Brazilian freshwater tropical lakes. Fisheries Management and Ecology, v. 11, p. 71-79, 2004.

LOWE, S.; BROWNE, M.; BOUDJELAS, S.; POORTER, M. de 100 of the World's Worst Invasive Alien Species A selection from the Global Invasive Species Database. Auckland, IUCN/SSC Invasive Species Specialist Group (ISSG), 2000.

MARTINS, D.; COSTA, N. V.; TERRA, M. A.; MARCHI, S. R. Caracterização da comunidade de plantas aquáticas de dezoito reservatórios pertencentes a cinco bacias hidrográficas do Estado de São Paulo. Planta Daninha, v. 26, n. 1, p. 17-32, 2008.

McNEELY, J. A. The Great Reshuffling: Human Dimensions of Invasive Alien Species. Gland: Switzerland and Cambridge, UK IUCN, 2001.

MILI, P. S. M.; TEIXEIRA, R. L. Notas ecológicas do bagre-africano Clarias gariepinus (Burchell, 1822) (Teleostei, Clariidae), de um córrego do sudeste do Brasil. Boletim do Museu de Biologia Mello Leitão, v. 19, p. 45-51, 2006.

MOREIRA FILHO, O.; BUCKUP, P. A. A poorly known case of watershed transposition between the São Francisco and upper Paraná River basins. Neotropical Ichthyology, v. 3, n. 3, p. 449-452, 2005.

NEVES, T.; FOLONI, L. L.; PITELLI, R. A. Controle químico do aguapé (Eichbornia crassipes). Planta Daninha, v. 20, p. 89-97, 2002.

OLIVEIRA, M. D.; TAKEDA, A. M.; BARROS, L. F.; BARBOSA, D. S.; RESENDE, E. K. Invasion by Limnoperna fortunei (Dunker, 1857) (Bivalvia, Mytilidae) of the Pantanal wetland, Brazil. Biological Invasions, v. 8, p. 97-104, 2006.

OPORTO, L. T. Modificações em longo prazo na ictiofauna da planície de inundação do alto rio Paraná, Brasil. 2008. 40f. Dissertação (Mestrado) Universidade Estadual de Maringá, Maringá, 2008.

PASTORINO, G.; DARRIGRAN, G. A.; MARTIN, S. M.; LUNASCHI, L. Limnoperna fortunei (Dunker, 1857) (Mytilidae), nuevo Bivalvo invasor en aguas del Río de la Plata. Neotropica, v. 39, n. 101-102, p. 34, 1993. 
POSEY, M. H.; WIGAND, C.; STEVENSON, J. C. Effects of an introduced aquatic plant, Hydrilla verticillata, on benthic communities in the Upper Chesapeake Bay. Estuarine Coastal Shelf Science, v. 37, n. 5, p. 539-555, 1993.

QUEROL, M. V. M.; QUEROL, E.; PESSANO, E. F. C.; AZEVEDO, C. L. de O. Ocorrência da carpa húngara, Cyprinus carpio (Linnaeus, 1758) e disseminação parasitária, no Arroio Felizardo, Bacia do médio Rio Uruguai, Uruguaiana, RS, Brasil. Biodiversidade Pampeana, v. 3, p. 21-23, 2005.

RAMOS, I. P.; ZANATTA, A. S.; ZICA, E. O. P.; SILVA, R. J.; CARVALHO, E. D. Impactos ambientais de pisciculturas em tanques-rede sobre águas continentais brasileiras: Revisão e Opinião. In: CYRINO, J. E. P.; URBINATI, E. C.; FRACALOSSI, D. M.; CASTAGNOLLI, N. (Org.) Tópicos especiais em piscicultura de água doce tropical intensiva. São Paulo: TecArt, 2005, p. 87-98.

RICHARDSON, D. M.; PYSEK, P. Classics in physical geography revisited. Elton, C. S. 1958: The ecology of invasions by animals and plants. Methuen: London. Progress in Physical Geography, v. 31, n. 6, p. 659-666, 2007.

RODRÍGUEZ, J. P. Exotic species introductions into South America: an underestimated threat? Biodiversity and Conservation, v. 10, p. 1983-1996, 2001.

SANTOS, J. G. A. da S.; LAMONICA, M. N. Água de lastro e bioinvasão: introdução de espécies exóticas associada ao processo de mundialização. Vértices, v. 10, n. 1-3, p. 141-152, 2008.

SAX, D. F.; BROWN, J. H. The paradox of invasion. Global Ecology and Biogeography, v. 9, n. 5, p. 363-371, 2000.

SHARP, R. L.; LARSON, L. R.; GREEN, G. T. Factors influencing public preferences for invasive alien species management. Biological Conservation, v. 144, p. 2097-2104, 2011.

SIMBERLOFF, D.; STILING, P. How risky is biological control? Ecology, v. 77, n. 7, p. 1965-1974, 1996. 
SIMÕES, N. R.; ROBERTSON, B. A.; LANSAC-TÔHA, F. A.; TAKAHASHI, E. M.; BONECKER, C. C., VELHO, L. F. M.; JOKO, C. Y. Exotic species of zooplankton in the Upper Paraná River floodplain, Daphnia lumboltzi Sars, 1885 (Crustacea: Branchiopoda). Brazilian Journal of Biology, v. 69, n. 2, p. 551-558, 2009.

SOUZA, R. C. C. L.; CALAZANS, S. H.; SILVA, E. P. Impacto das espécies invasoras no ambiente aquático. Ciência e Cultura, v. 61, n. 1, p. 35-41, 2009.

THOMAZ, S. M. Fatores ecológicos associados à colonização e desenvolvimento de macrófitas aquáticas e desafios de manejo. Planta Daninha, v. 20, p. 13-25, 2002.

THOMAZ, S. M.; CARVALHO, P.; PADIAL, A. A.; KOBAYASHI, J. T. Temporal and spatial patterns of aquatic macrophyte diversity in the Upper Paraná River floodplain. Brazilian Journal of Biolog y, v. 69, n. 2, p. 617-625, 2009a.

THOMAZ, S .M.; CARVALHO, P.; MORMUL, R. P.; FERREIRA, F. A.; SILVEIRA, M. J.; MICHELAN, T. S. Temporal trends and effects of diversity on occurrence of exotic macrophytes in a large reservoir. Acta Oecologica, v. 35, p. 614-620, 2009b.

TORGAN, L. C.; SALOMONI, S. E.; BICCA, A. A. Diatomáceas sobre Limnoperna fortunei (Dunker), molusco introduzido no lago Guaíba, Sul do Brasil. Revista Brasileira de Botânica, v. 32, n. 1, p. 23-31, 2009.

ULIANO-SILVA, M.; FERNANDES, F. F. C. F.; HOLANDA, I. B. B. de; REBELO, M. F. Invasive species as a threat to biodiversity: The golden mussel Limnoperna fortune approaching the Amazon River basin. In: ALLODI, S. (Org.). Exploring Themes on Aquatic Toxicolog y. Research Signpost, Trivandrum, Kerala, 2013. p. 135-148.

VITOUSEK, P. M.; D’ ANTONIO, C. M.; LOOPE, L. L.; REJMÁNEK, M.; WESTBROOKS, R. Introduced species: A significant component of human-caused global change. New Zealand Journal of Ecology, v. 21, n. 1, p. 1-16, 1997.

VITULE, J. R. S.; UMBRIA, S. C.; ARANHA, J. M. R. Introduction of the African catfish Clarias gariepinus (BURCHELL, 1822) into Southern Brazil. Biological Invasions, v. 8, p. 677-681, 2006. 
VITULE, J. R. S.; FREIRE, C. A.; SIMBERLOFF, D. Introduction of non-native freshwater fish can certainly be bad. Fish and Fisheries, v. 10, p. 98-108, 2009.

VITULE, J. R. S.; SKORA, F.; ABILHOA, V. Homogenization of freshwater fish faunas after the elimination of a natural barrier by a dam in Neotropics. Diversity and Distributions, v. 18, p. 111-120, 2012.

WHITFIELD, P. E.; GARDNER, T.; VIVES, S. P.; GILLIGAN, M. R.; COURTENAY JR, W. R.; RAY, G. C.; HARE, J. A. Biological invasion of the Indo-Pacific lionfish Pterois volitans along the Atlantic coast of North America. Marine Ecology Progress Series, v. 235, p. 289-297, 2002.

WHITTINGTON, I. D.; CHISHOLM, L. A. Diseases caused by Monogenea. In: EIRAS, J. C.; SEGNER, H.; WAHLI, T.; KAPOOR, B. G. (Org.) Fish Diseases. Enfield, NH, Science Publishers, 2008. Vol. 2. p. 682-816.

WITTE, F.; GOLDSCHMIDT, T.; WANINK, J.; Van OIJEN, M.; GOUDSWAARD, K.; WITTE-MAAS, E.; BOUTON, N. The destruction of an endemic species flock: quantitative data on the decline of the haplochromine cichlids of Lake Victoria. Environmental Biology of Fishes, v. 34, p. 1-28, 1992.

WITTENBERG, R.; COCK, M. J. W. Invasive alien species. How to address one of the greatest threats to biodiversity: a toolkit of best prevention and management practices. Wallingford, Oxon: CAB International, 2001.

Recebido em: 17 de setembro de 2014 Aceito em: 29 de junho de 2015 